Can the crystallization rate be independent from the crystallization enthalpy? The case of amorphous silicon

This content has been downloaded from IOPscience. Please scroll down to see the full text.

2012 J. Phys.: Condens. Matter 24095401

(http://iopscience.iop.org/0953-8984/24/9/095401)

View the table of contents for this issue, or go to the journal homepage for more

Download details:

IP Address: 161.116.168.74

This content was downloaded on 17/01/2014 at 09:09

Please note that terms and conditions apply. 


\title{
Can the crystallization rate be independent from the crystallization enthalpy? The case of amorphous silicon
}

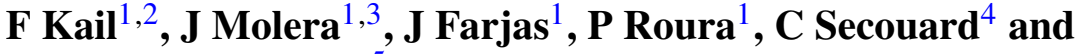 \\ P Roca i Cabarrocas ${ }^{5}$ \\ ${ }^{1}$ GRMT, Department of Physics, University of Girona, Montilivi Campus, E17071-Girona, Catalonia, \\ Spain \\ ${ }^{2}$ Department of Fisica Aplicada and Optica, University of Barcelona, E08028-Barcelona, Catalonia, \\ Spain \\ ${ }^{3}$ Escola Politecnica Superior (EPS), GRTD, University of Vic, E08500-Vic, Catalonia, Spain \\ ${ }^{4}$ CEA Grenoble, LTS, 17 rue des Martyrs, 38054 Grenoble cedex, France \\ ${ }^{5}$ LPICM, Ecole Polytechnique, 91128 Palaiseau, France \\ E-mail: pere.roura@udg.es
}

Received 22 November 2011, in final form 11 January 2012

Published 9 February 2012

Online at stacks.iop.org/JPhysCM/24/095401

\begin{abstract}
The crystallization enthalpy measured in a large series of amorphous silicon (a-Si) materials varies within a factor of 2 from sample to sample (Kail et al 2011 Phys. Status Solidi RRL 5 361). According to the classical theory of nucleation, this variation should produce large differences in the crystallization kinetics leading to crystallization temperatures and activation energies exceeding $550^{\circ} \mathrm{C}$ and $1.7 \mathrm{eV}$, respectively, the 'standard' values measured for a-Si obtained by self-implantation. In contrast, the observed crystallization kinetics is very similar for all the samples studied and has no correlation with the crystallization enthalpy. This discrepancy has led us to propose that crystallization in a-Si begins in microscopic domains that are almost identical in all samples, independently of their crystallization enthalpy. Probably the existence of microscopic inhomogeneities also plays a crucial role in the crystallization kinetics of other amorphous materials and glasses.
\end{abstract}

\section{Introduction}

The crystallization of amorphous silicon (a-Si) is driven by the reduction of its free energy when it becomes crystalline, $\Delta g_{\mathrm{c}}$ (the crystallization free energy). Like most solid-state transformations, crystallization is a heterogeneous transformation governed by thermally activated nucleation and growth steps. Transmission electron microscopy allows the nucleation, $r_{\mathrm{n}}$, and growth rates, $r_{\mathrm{g}}$, to be measured by counting the number of crystallites that appear per unit time and by measuring their size, respectively. For pure substances, $r_{\mathrm{n}}$ and $r_{\mathrm{g}}$ follow an Arrhenius dependence on temperature [1-3]:

$$
\begin{gathered}
r_{\mathrm{n}}=r_{\mathrm{n} 0} \exp \left(-\Delta E_{\mathrm{n}} / R T\right) \quad \text { and } \\
r_{\mathrm{g}}=r_{\mathrm{g} 0} \exp \left(-\Delta E_{\mathrm{g}} / R T\right)
\end{gathered}
$$

where $\Delta E_{\mathrm{n}}\left(\Delta E_{\mathrm{g}}\right)$ is the activation energy for nucleation (growth), $r_{\mathrm{n} 0}\left(r_{\mathrm{g} 0}\right)$ the pre-exponential nucleation (growth) rate constant, $T$ the temperature, and $R$ the Boltzmann constant. Most isothermal experiments reveal the existence of an incubation time, $t_{\text {inc }}$, required before any crystallite can be observed $[4,1,5]$.

Detailed studies for determining the values of the kinetic parameters $\left(r_{\mathrm{n}}, r_{\mathrm{g}}\right.$, and $\left.t_{\mathrm{inc}}\right)$ have been done on a-Si obtained after ion implantation and consistent values have been obtained by independent authors [4, 1]. They are detailed in table 1 and will be referred to as the 'standard' (sd) crystallization parameters. In contrast, hydrogenated a-Si films obtained by deposition from a vapor phase usually crystallize at higher rates [5] with nucleation and growth rates that depend on the particular film.

Despite the technological interest in elucidating the structural parameters that have an influence on the 
Table 1. Standard crystallization parameters of a-Si [5]. $r_{i 0}$ : pre-exponential constants for nucleation $(i=\mathrm{n})$, growth $(i=\mathrm{g})$ and crystallization $(i=\mathrm{c})$ rates. $\Delta E_{i}$ : activation energies; $\Delta h_{\mathrm{c}}$ : the enthalpy of crystallization.

\begin{tabular}{cc}
\hline$r_{\mathrm{n} 0}=1.7 \pm 0.4 \times 10^{26} \mu \mathrm{m}^{-3} \mathrm{~s}^{-1}$ & $\Delta E_{\mathrm{n}}=5.30 \pm 0.10 \mathrm{eV}$ \\
$r_{\mathrm{g} 0}=2.1 \pm 0.4 \times 10^{13} \mu \mathrm{m} \mathrm{s}^{-1}$ & $\Delta E_{\mathrm{g}}=3.10 \pm 0.10 \mathrm{eV}$ \\
$r_{\mathrm{c} 0}=3.6 \pm 0.4 \times 10^{16} \mathrm{~s}^{-1}$ & $\Delta E_{\mathrm{c}}(3 \mathrm{D})=3.65 \pm 0.10 \mathrm{eV}$ \\
\multicolumn{2}{c}{$\Delta h_{\mathrm{c}}=423 \mathrm{~J} \mathrm{~g}^{-1}(0.10 \mathrm{eV} /$ atom $)$} \\
\hline
\end{tabular}

crystallization rate (for instance, one would like to reduce the nucleation rate and increase the crystallization rate in order to produce large grain polycrystalline silicon films), that relationship remains obscure. Analysis by means of Raman spectroscopy of films annealed near the end of the incubation period has shown that there is no relationship between $r_{\mathrm{n}}$ and $r_{\mathrm{g}}$ in the bond-angle strain [6]. It has been concluded that the varying density of dangling bonds left behind by dehydrogenation during the heating ramp to the crystallization temperature cannot have an appreciable effect on nucleation [7, 8] and that film microstructure (such as microvoid density) does not correlate with the incubation time [9]. The most appealing explanation for the systematically shorter incubation time for films deposited by hot wire chemical vapor deposition (HWCVD) compared with plasma-enhanced CVD (PECVD) films is that the tendency of hydrogen to get clustered in HWCVD films would leave larger regions with a higher medium-range order [8]. It is argued that nucleation would be easier in these regions.

We have recently shown [10] that the crystallization enthalpy of a-Si varies within a factor of 2 for a large set of a-Si materials grown by different techniques. Since the classical theory of nucleation states that both nucleation and growth depend on the free energy of the amorphous state [1], one would expect a clear dependence of the crystallization rate on the crystallization enthalpy. Remember that

$$
\Delta g_{\mathrm{c}} \equiv \Delta h_{\mathrm{c}}-T \Delta s_{\mathrm{c}}
$$

where $\Delta s_{\mathrm{c}}$ is the entropy of crystallization. Therefore, we wondered whether we could find an experimental correlation that would explain the observed variability of the kinetic parameters governing a-Si crystallization.

This introduction is followed by a section in which the functional dependences of $r_{\mathrm{n}}$ and $r_{\mathrm{g}}$ on the free energy (or enthalpy, through equation (2)) of the amorphous state are made explicit and compared with experimental results from the literature. Then, the experimental results for a-Si are collected and we show that there is no correlation between the crystallization rate and the crystallization enthalpy. This negative result is further highlighted in section 3 where it is confirmed that the expected variation of the kinetic constants is much larger than the observed variability. This discrepancy between theory and experiment is then discussed in terms of structural inhomogeneity in a-Si. Finally, we conclude with a brief summary.

\section{Theoretical dependences and a literature review}

The critical step for crystallization to begin is the formation of a nucleus, i.e., a crystalline cluster large enough that

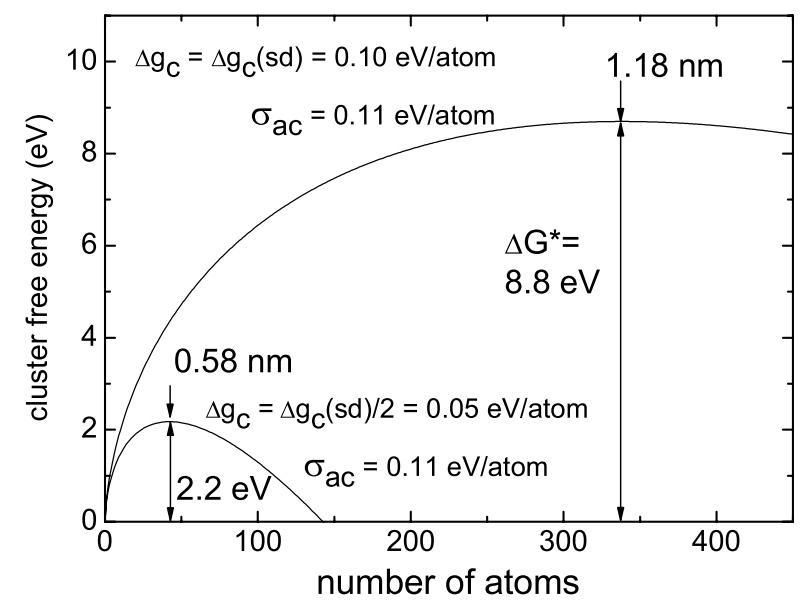

Figure 1. Free energy of the crystalline clusters for two different values of the free energy of crystallization. The critical cluster sizes are indicated as well as the nucleation barrier, $\Delta G^{*}$. The lower curve corresponds to the barrier for the standard kinetics.

the addition of one atom from the amorphous surrounding phase produces a diminution of the free energy of the system (figure 1). Beyond this critical size, the energy of the a-c (amorphous-crystalline) interface increases more slowly than the diminution of the energy of the bulk atoms in the cluster. The energy corresponding to this critical size is known as the 'nucleation barrier', $\Delta G^{*}$. It depends on the free energy of crystallization, $\Delta g_{\mathrm{c}}$, and the a-c surface energy, $\sigma_{\mathrm{ac}}$, according to $[1,11]$ :

$$
\Delta G^{*}=\frac{16 \pi}{3} \frac{\sigma_{\mathrm{ac}}^{3}}{\Delta g_{\mathrm{c}}^{2}} .
$$

The clusters above the critical size grow continuously and develop a population of crystallites with different sizes. After a transient period (lasting $t_{\text {inc }}$ ), a steady-state population of nuclei is achieved leading to the formation at a constant rate of crystallites large enough to be observable, i.e., the (steady-state) nucleation rate $r_{\mathrm{n}}$ of equation (1). Its activation energy can be decomposed into three components [1]:

$$
\Delta E_{\mathrm{n}}=\Delta G^{*}+\Delta G_{\mathrm{A}}+\Delta G_{\mathrm{f}},
$$

where $\Delta G_{\mathrm{f}}$ is the formation energy of the structural defects that promote the atomic jumps across the a-c interface and $\Delta G_{\mathrm{A}}$ is the activation energy for the jumps measured from the amorphous side (figure 2). In principle, $\Delta G_{\mathrm{f}}$ can be considered independent from $\Delta g_{\mathrm{c}}$, whereas a weak dependence would be expected for $\Delta G_{\mathrm{A}}$. Among all the paths leading an atom from the amorphous to the crystalline side of the $a-c$ interface, there is one path with the least maximum 


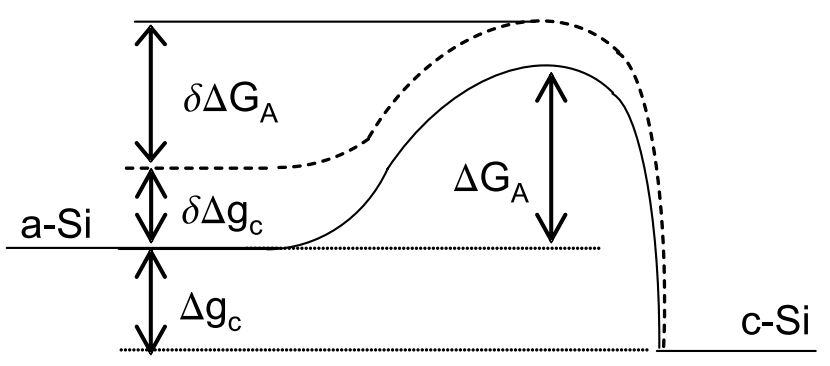

Figure 2. Variation of the free energy diagram across the a-c interface following an increment of the crystallization free energy, $\delta \Delta g_{\mathrm{c}}$. The diminution of the barrier for atomic jumps $\left(\delta \Delta G_{\mathrm{A}}\right)$ is presumably lower than $\delta \Delta g_{\mathrm{c}}$

free energy. $\Delta G_{\mathrm{A}}$ corresponds to the microscopic state at the maximum of this path (the 'activated complex' [12]). Since the activated complex lies between the amorphous and crystalline phases, it has been argued that its energy would experience a smaller variation than that of the amorphous phase, $\delta \Delta g_{\mathrm{c}}$ [13] (figure 2), i.e.,

$$
\left|\delta \Delta G_{\mathrm{A}}\right|<\left|\delta \Delta g_{\mathrm{c}}\right|
$$

As regards the pre-exponential term of $r_{\mathrm{n}}$, its dependence on $\Delta g_{\mathrm{c}}$ is given by $[1,11]$

$$
r_{\mathrm{n} 0}=C \frac{\Delta g_{\mathrm{c}}}{\sqrt{\sigma_{\mathrm{ac}}}}
$$

where the constant $C$ collects together several parameters related to the atomic density in $\mathrm{a}-\mathrm{Si}$, the attempt frequency and a weak temperature dependence $(1 / \sqrt{k T})$.

The radii of the observable crystallites grow at a constant rate equal to the growth rate, $r_{\mathrm{g}}$, which depends exclusively on the balance of the atomic jumps from both sides of the a-c interface, so neither the pre-exponential constant nor the activation energy depends on $\sigma_{\mathrm{ac}}$. The activation energy, $\Delta E_{\mathrm{g}}$, retains a weak dependence on $\Delta g_{\mathrm{c}}$ through $\Delta G_{\mathrm{A}}[1]$

$$
\Delta E_{\mathrm{g}}=\Delta G_{\mathrm{A}}+\Delta G_{\mathrm{f}},
$$

whereas the dependence of the pre-exponential rate constant can be made explicit:

$$
r_{\mathrm{g} 0}=C^{\prime}\left[1-\exp \frac{-\Delta g_{\mathrm{c}}}{R T}\right] .
$$

This expression generalizes that of [1] for arbitrary values of $\Delta g_{\mathrm{c}}$.

From equations (3) and (5)-(8), it is clear that an increment of $\Delta g_{\mathrm{c}}$ will lead to a faster crystallization kinetics mainly by reducing the nucleation barrier and, to a lesser extent, by reducing the barrier for atomic jumping across the a-c interface $\left(\Delta G_{\mathrm{A}}\right)$ and incrementing the pre-exponential constants. The inverse applies for a reduction of $\Delta g_{\mathrm{c}}$.

A survey of the literature devoted to the crystallization of amorphous materials and glasses shows that the theoretical dependence on the crystallization enthalpy $\left(\Delta h_{\mathrm{c}}\right)$ is not considered suspect even though experiments are unable to reveal it. Several examples follow. Amorphous calcium carbonate powders obtained by precipitation from aqueous solutions crystallize within different ranges of $\Delta h_{\mathrm{c}}$ depending on the report: 12.26-50.7 [14], 13.1-19.1 [15] and 12.8-15.4 kJ mol ${ }^{-1}$ [16]. Amorphous Se [17, 18] and $\mathrm{GeSe}_{2}$ [18] have $\Delta h_{\mathrm{c}}$ values that differ by as much as $40 \%$ when obtained by ball milling or by rapid quenching of the liquid. For most of these cases large variations of the crystallization temperature or activation energy (see equations (9)-(10)) are reported; however, the correlation with $\Delta h_{\mathrm{c}}$ (if any) is often opposite to what is expected [18, 17, 15]. Multicomponent chalcogenide glasses $\left(\mathrm{Ga}_{15} \mathrm{Se}_{85-x} \mathrm{Ag}_{x}\right.$ [19], $\mathrm{Se}_{80-x} \mathrm{Te}_{20} \mathrm{Ag}_{x}$ [20]) crystallize in a narrow range of temperatures not exceeding $10^{\circ} \mathrm{C}$ despite the very large variation of $\Delta h_{\mathrm{c}}$ of one order of magnitude [20]. In these materials, several well-known facts could hide the expected dependence on $\Delta h_{\mathrm{c}}$. For instance, if the resulting material is nanocrystalline, the crystallization enthalpy has to be corrected by the energy of the grain boundaries to obtain the enthalpy of the amorphous state [21]. On the other hand, in multicomponent glasses the crystallization kinetics can be controlled by atomic diffusion.

None of these effects applies to a-Si because of its high chemical purity and because it crystallizes in a polycrystalline microstructure with grain sizes larger than $100 \mathrm{~nm}$ [5]. This is to say that, in principle, a-Si seems to be a good candidate for testing the expected dependences on the crystallization enthalpy. This exercise is worth doing because we have not found, in the literature, any attempt to experimentally verify the expected dependences of the crystallization of amorphous materials or glasses on the crystallization enthalpy.

Note that once $\Delta E_{\mathrm{g}}$ and $\Delta E_{\mathrm{n}}$ are known, the nucleation barrier $\Delta G^{*}$ can be obtained through subtraction (equations (4) and (8)). From the 'standard values' of table $1, \Delta G^{*}=$ $2.2 \pm 0.2 \mathrm{eV}$ for a-Si. The crystallization enthalpy of a-Si amorphized by ion implantation was measured with accuracy and falls within a narrow range of $\Delta h_{\mathrm{c}}=423 \pm 25 \mathrm{~J} \mathrm{~g}^{-1}$ $(0.12 \pm 0.01 \mathrm{eV} /$ atom $)$ [22]. Since the heat capacity of a-Si has been recently measured [23], we can obtain the crystallization free energy of a-Si at the typical crystallization temperature of $1000 \mathrm{~K}, \Delta g_{\mathrm{c}}=0.10 \pm 0.01 \mathrm{eV} /$ atom. Finally, equation (3) can be applied to determine the surface energy, $\sigma_{\mathrm{ac}}=0.11 \mathrm{eV} /$ atom. From these values, the free energy of formation of crystalline clusters has been calculated for this 'standard' a-Si (figure 1).

\section{Experimental results for a-Si}

For around twenty different a-Si samples, the crystallization has been analyzed using differential scanning calorimetry (DSC). The samples where grown by HWCVD, PECVD and electron beam evaporation (EBE). This last technique gave pure a-Si, whereas hydrogenated a-Si $(\mathrm{a}-\mathrm{Si}: \mathrm{H})$ was obtained with the other two techniques. In the case of PECVD, in addition to conventional a-Si:H, some films were obtained in plasma conditions such that nanocrystals as well as $\mathrm{SiH}_{x}$ radicals were deposited on the substrate, resulting in a special grade of amorphous silicon known as polymorphous silicon (pm-Si:H) [24]. All films were several microns thick, so 


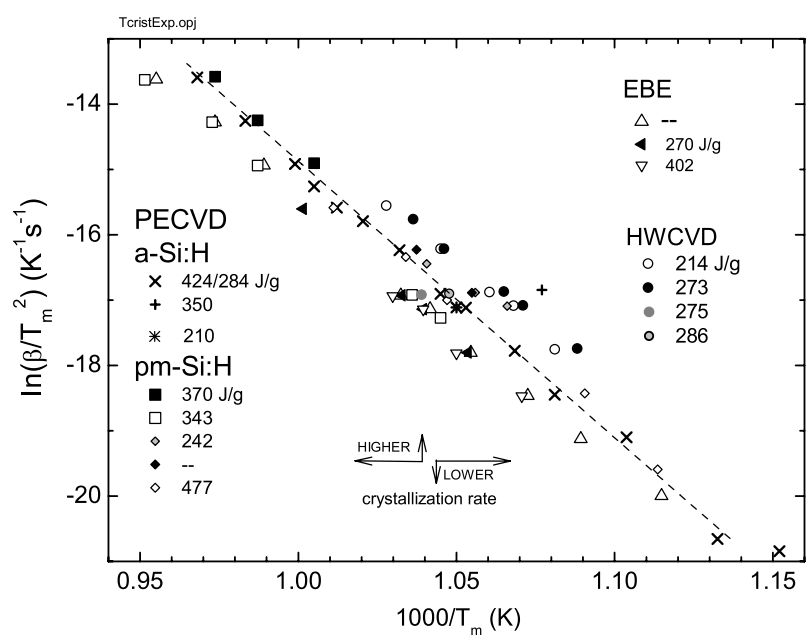

Figure 3. Kissinger plot for the DSC crystallization peak for several samples with varying values of the crystallization enthalpy (quoted next to the symbols). The line is not a fitting, but the expected dependence for the 'standard kinetics'; see table 1.

heterogeneous nucleation at the film surfaces can be neglected except for one sample that consisted of a $0.5 \mu \mathrm{m}$ thin a-Si:H film deposited on a highly crystalline 'microcrystalline film' $(\mu \mathrm{c}-\mathrm{Si}: \mathrm{H})$ of the same thickness. Further details about these samples and the DSC experiments have been published elsewhere [25].

When crystallization is produced during thermal treatment carried out at a constant temperature rise ( $\beta=\mathrm{d} T / \mathrm{d} t=$ constant), its kinetics can still be described by the Kolmogorov-Johnson-Mehl-Avrami (KJMA) model [26-30], slightly modified by a constant factor multiplying $r_{\mathrm{n} 0}$ and $r_{\mathrm{g} 0}$ that depends on the activation energies and the kind of growth [31]. For a 3D growth of the crystallites in a-Si, this factor is 0.87 . We have verified for selected samples that the DSC crystallization peak has the shape expected for a KJMA kinetics [32]. Therefore, the crystallization rate, $r_{\mathrm{c}}$ :

$$
r_{\mathrm{c}}=\left(r_{\mathrm{n}} r_{\mathrm{g}}^{3}\right)^{1 / 4} \equiv r_{\mathrm{c} 0} \mathrm{e}^{-\Delta E_{\mathrm{c}} / R T},
$$

where

$$
\begin{aligned}
& r_{\mathrm{c} 0}=\left(r_{\mathrm{n} 0} r_{\mathrm{g} 0}^{3}\right)^{1 / 4} \quad \text { and } \\
& \Delta E_{\mathrm{c}}=\left(\Delta E_{\mathrm{n}}+3 \Delta E_{\mathrm{g}}\right) / 4,
\end{aligned}
$$

can be obtained from the DSC experiments. The dependence of the DSC peak temperature, $T_{\mathrm{m}}$, on the heating rate, $\beta$, is related through the Kissinger equation [33, 34]:

$$
\ln \frac{\beta}{T_{\mathrm{m}}^{2}}=-\frac{\Delta E_{\mathrm{c}}}{R T_{\mathrm{m}}}+\ln \frac{R r_{\mathrm{c} 0} 0.87}{\Delta E_{\mathrm{c}}} .
$$

Therefore, $r_{\mathrm{c} 0}$ and $\Delta E_{\mathrm{c}}$ can be obtained from a linear fitting to the Kissinger plot $\left(\ln \beta / T_{\mathrm{m}}^{2}\right.$ versus $\left.1000 / T_{\mathrm{m}}\right)$ of the experimental points. On the other hand, the crystallization enthalpy, $\Delta h_{\mathrm{c}}$, has been obtained from the area under the crystallization peak, once it has been corrected for the crystalline fractions before and after the DSC peak [10].

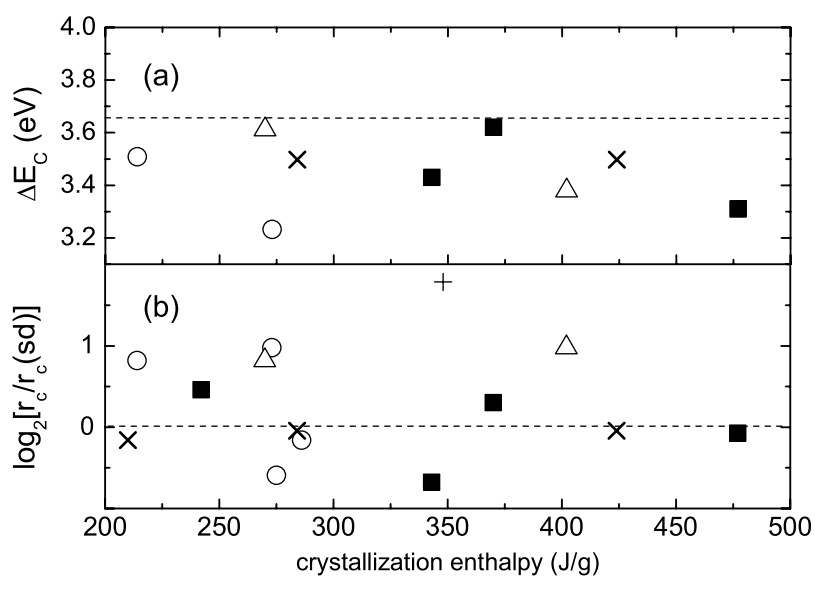

Figure 4. The lack of any correlation between the activation energy or the crystallization rate at $950 \mathrm{~K}$ and the crystallization enthalpy is evident from parts (a) and (b), respectively. Crosses are for PECVD a-Si:H, squares for PECVD pm-Si:H, circles for HWCVD, and triangles for EBE. Horizontal lines: standard values.

The Kissinger plot collecting the DSC results obtained for all the samples is shown in figure 3, where the heating rate spans over more than three orders of magnitude (from 0.02 to $40 \mathrm{~K} \mathrm{~min}^{-1}$ ). Together with the experimental points, the straight line represents the $\beta / T_{\mathrm{m}}$ dependence predicted from the standard kinetic parameters (table 1). The points of any sample tend to be aligned close to this reference line, indicating that their activation energy is similar to that of the standard kinetics $\left(\Delta E_{\mathrm{c}}(\mathrm{sd})=3.65 \mathrm{eV}\right)$ and that the crystallization rate is slightly higher or lower depending on whether they fall on the right or on the left of the reference line, respectively.

The crystallization enthalpy of most samples is also quoted in figure 3. Notice that most of them have values lower than that of a-Si obtained by ion implantation $\left(423 \mathrm{~J} \mathrm{~g}^{-1}\right.$; see table 1). A quick inspection seems to show that there is no correlation between the crystallization kinetic parameters and $\Delta h_{\mathrm{c}}$. This fact is made clearer in figure 4 , where the activation energy and the crystallization rate (equation (9)) are plotted versus $\Delta h_{\mathrm{c}}$

The activation energy is lower for all samples than the standard activation energy (figure 4(a)). Apart from this general feature, no trend is observed, as if $\Delta E_{\mathrm{c}}$ were independent of $\Delta h_{\mathrm{c}}$.

The crystallization rate can be obtained through the values of the peak temperature, $T_{\mathrm{m}}$, and $\Delta E_{\mathrm{c}}$. Rearrangement of the Kissinger equation (equation (11)) leads to

$$
r_{\mathrm{c}}\left(T_{\mathrm{m}}\right) \equiv r_{\mathrm{c} 0} \exp \frac{-\Delta E_{\mathrm{c}}}{R T_{\mathrm{m}}}=\beta \frac{\Delta E_{\mathrm{c}}}{0.87 R T_{\mathrm{m}}^{2}} .
$$

In figure 4(b), the values of $r_{\mathrm{c}}\left(T_{\mathrm{m}}\right)$ relative to the standard crystallization rate at $950 \mathrm{~K}$ have been plotted versus $\Delta h_{\mathrm{c}}$. All the experimental points, except that for the a-Si:H thin film grown on crystalline $\mathrm{Si}$ (symbol + in figures 3 and 4(b)), vary around the standard value within a factor of $2\left(r_{\mathrm{c}}(\mathrm{sd}) / 2<\right.$ $\left.r_{\mathrm{c}}<2 r_{\mathrm{c}}(\mathrm{sd})\right)$. Probably the greater crystallization rate of the a-Si:H thin film is due to some degree of epitaxial growth on 
the crystalline substrate [35]. Anyway, the ensemble of points shows that there is no correlation of $r_{\mathrm{c}}$ with $\Delta h_{\mathrm{c}}$.

We wish to focus the reader's attention on one particularly significant PECVD a-Si:H sample (cross symbols in figure 3). It was measured by DSC after deposition and after being stored for five years at room temperature. After this long period of time its crystallization enthalpy had diminished from 424 to $284 \mathrm{~J} \mathrm{~g}^{-1}$ [10] but the kinetic parameters remained the same. The last measurement that we did corresponded to the four points at the highest heating rates (upper left cross points in figure 3). Notice that, within the experimental accuracy, they are well aligned with the points measured at lower rates just after sample deposition. For this particular sample, we can state that, after a $35 \%$ reduction of $\Delta h_{\mathrm{c}}$, the crystallization rate remained the same within an error bar of $\pm 10 \%$.

Complementary experiments where the growth rate has been found to be independent of the amorphous state enthalpy have been reported by other authors [13]. In this case, the epitaxial crystallization rate of a $2 \mu \mathrm{m}$ thick amorphous region obtained by ion implantation on a c-Si wafer remained essentially the same (within an error bar of $3 \%$ ) before and after partial structural relaxation. From the Raman spectra of that sample, we can estimate [36] that the crystallization enthalpy diminished from 812 to $605 \mathrm{~J} \mathrm{~g}^{-1}$ (i.e., by $60 \mathrm{meV} /$ atom) due to structural relaxation.

\section{Discussion}

We begin this section by predicting, according to the classical theory, how much the crystallization rate and activation energy should vary for the samples studied. The crystallization enthalpy of the reference a-Si (table 1) falls in the upper range of the measured values (figure 3) and it is twice their minimum value. Since the entropy makes a minor contribution to the free energy of a-Si, $\Delta g_{\mathrm{c}}$ (equation (2)), at the crystallization temperature of our experiments (section 2), we will consider, for simplicity, that the free energy of our samples varies from $\Delta g_{\mathrm{c}}(\mathrm{sd})$ to $\Delta g_{\mathrm{c}}(\mathrm{sd}) / 2$. In figure 5 we have plotted the expected $\beta / T_{\mathrm{m}}$ dependence for $\Delta g_{\mathrm{c}}(\mathrm{sd}) / 2$ as a solid line. It has been calculated by supposing that both the nucleation and growth rates depend on $\Delta g_{\mathrm{c}}$ as predicted by the classical theory of crystallization (section 2). Additionally, the dashed line in figure 5 corresponds to the dependence that would be expected if only the nucleation rate were affected by the change from $\Delta g_{\mathrm{c}}(\mathrm{sd})$ to $\Delta g_{\mathrm{c}}(\mathrm{sd}) / 2$.

According to this prediction, the crystallization temperature should vary from sample to sample by as much as $550^{\circ} \mathrm{C}$ and the influence of $\Delta g_{\mathrm{c}}$ on the nucleation process produces most of this variation. However, the experimental variation is less than $10 \%$ of this value and, as seen in section 3, it does not correlate with the crystallization enthalpy. In figure 5, the expected activation energy is also quoted. Its value, $5.30 \mathrm{eV}$, is very far from the range of experimental values (figure 4(a)). Therefore, a serious discrepancy arises between the classical theory of nucleation and the experiments. The challenge is finding how this discrepancy can be solved.

On the one hand, given the large influence of $\Delta g_{\mathrm{c}}$ on the nucleation barrier (equation (3) and figure 1), a

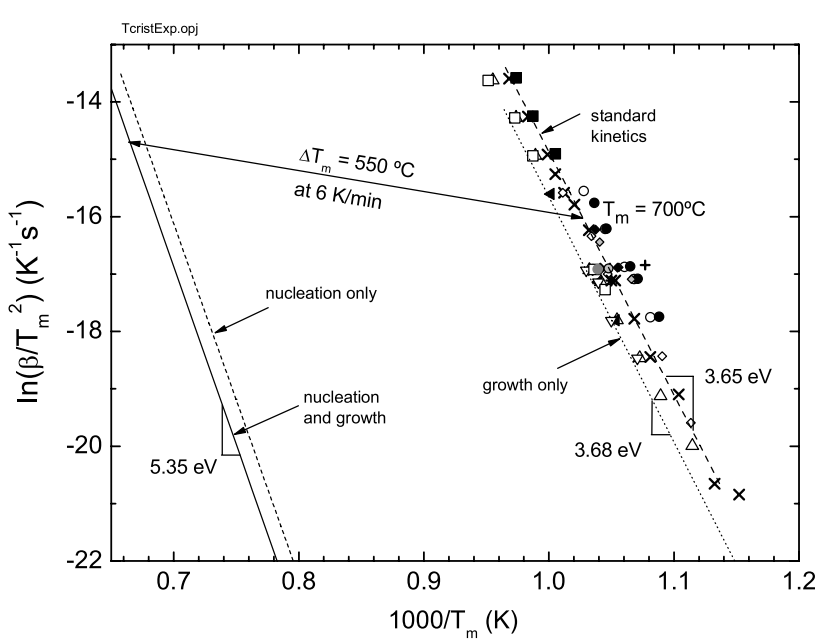

Figure 5. The predicted Kissinger plot for a reduction of the crystallization enthalpy when it affects the nucleation and growth rates (solid line), the nucleation rate only (dashed) and the growth rate only (dotted).

parallel variation of the a-c interfacial energy, $\sigma_{\mathrm{ac}}$, that would exactly compensate for the $\Delta g_{\mathrm{c}}$ variations is very unlikely. On the other hand, this constancy of kinetic parameters and its independence from the crystallization enthalpy suggest that nuclei must appear in very similar local environments (virtually identical) for all the samples despite the very different enthalpies (averaged over the material volume) delivered by DSC. Therefore, it seems natural that understanding the experimental crystallization rates requires that the inhomogeneous nature of the a-Si microstructure be considered.

This kind of explanation was recently given to interpret why the incubation period was shorter in HWCVD films with low $\mathrm{H}$ content than in conventional PECVD films [8]. According to that research, nucleation in both kinds of films would occur in $\mathrm{H}$-free regions, which would be better ordered. These nucleation zones could also be the result of the inhomogeneous spatial distribution of structural defects. In fact, in our recent paper devoted to quantifying $\Delta h_{\mathrm{c}}$ [10], we proposed that the excess of enthalpy above the minimum value common for all deposition techniques was due to different densities of structural defects. Nucleation could occur in those better ordered regions, free of defects. To be more precise, these regions would have maximum values of short-range and medium-range order.

To validate this explanation, one should explain why both the local free energy and the a-c surface energy are identical for different samples. Experimental results strongly suggest that both the short-range order (SRO) [25] and the medium-range order (MRO) [37] tend to reach a maximum value at the onset of crystallization. The SRO is mainly related to bond-angle strain, which can be quantified by Raman spectroscopy. After thermal relaxation up to the crystallization temperature, all a-Si materials (pure or hydrogenated) tend to a common value of bond-angle strain [6] that has been interpreted as experimental evidence of the configuration gap predicted in the 1980s for a-Si [38]. This value corresponds 


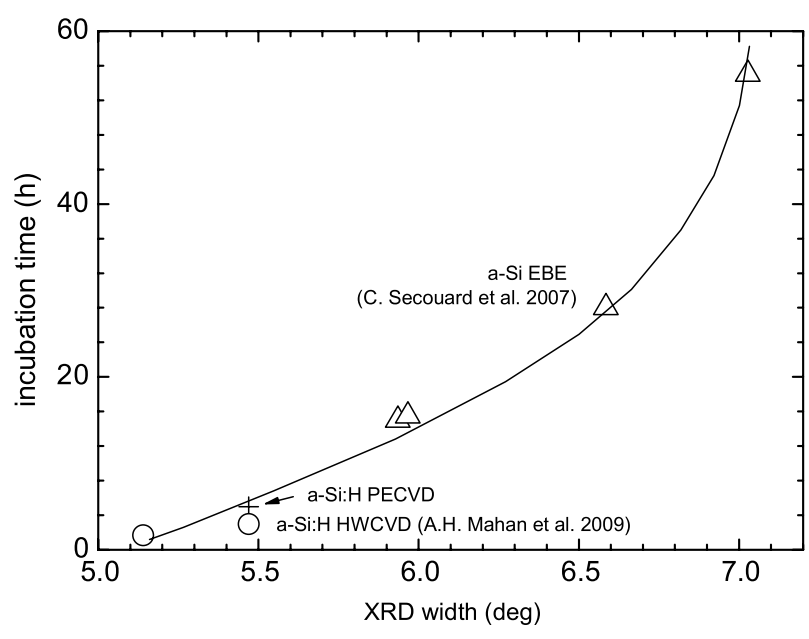

Figure 6. Correlation between the incubation time and the MRO quantified through the full width at half-maximum (FWHM) of the first XRD diffraction peak ( $\mathrm{Cu}$ X-ray source). The line is a guide for the eye.

to a minimum value of the crystallization enthalpy around $240 \mathrm{~J} \mathrm{~g}^{-1}$ (the configurational energy gap between a-Si and c-Si) [10] that would determine the $\Delta g_{\mathrm{c}}$ value of the defect-free microscopic regions where nucleation occurs.

As regards the MRO, it has been shown that a-Si:H films deposited on the 'onset of crystallinity' have a maximum value of the correlation length quantified by the width of the first diffraction peak of the XRD curves [37]. Our own experiments on EBE [39] and PECVD samples show that, just before any crystalline fraction appears in the XRD curves, the width of the first diffraction peak tends to a common minimum value (maximum MRO) that coincides with that of [37]. Furthermore, a nice correlation can be established between the initial MRO (before annealing) and the incubation time, $t_{\text {inc }}$ (figure 6): samples having a broader first diffraction peak crystallize after longer incubation periods (this correlation was not clear in [8] because the MRO approached its maximum value there). Since the interfacial energy, $\sigma_{\mathrm{ac}}$, has an important effect on the nucleation barrier (equation (3)) and we have verified [39] that the SRO does not change during the incubation period (i.e., $\Delta g_{\mathrm{c}}$ remains constant), we propose that $\sigma_{\mathrm{ac}}$ is related to the MRO and that it is lower for higher MRO. This proposal is consistent with MRO being related to the topology of the $\mathrm{Si}-\mathrm{Si}$ covalent network [40].

An independent quantification of MRO in a-Si is achieved by fluctuation electron microscopy (FEM) [41]. These experiments are interpreted in terms of variable densities of 'paracrystals' that are highly distorted nanodomains retaining the crystalline topology. Although no systematic experiments have been done to correlate the MRO obtained by FEM and by XRD, it has been shown that the MRO of PECVD pm-Si:H films quantified by both techniques was higher than that of PECVD a-Si:H films [42, 43, 41]. If confirmed by complementary experiments, this correlation could explain why an amorphous region with higher MRO (larger density of paracrystals) would have a lower $\sigma_{\mathrm{ac}}$.

In summary, we propose that the discrepancy between the expected strong dependence of the crystallization rate on $\Delta h_{\mathrm{c}}$ and the lack of any experimental correlation can be explained by considering the heterogeneous structure of a-Si. Nucleation will begin in those regions free of defects having minimum values of $\sigma_{\mathrm{ac}}$ and $\Delta g_{\mathrm{c}} . \sigma_{\mathrm{ac}}$ is related to the local MRO whereas, in the absence of defects, $\Delta g_{\mathrm{c}}$ is related to the local SRO.

Once we have explained why the nucleation rate can be independent of the measured crystallization enthalpy, the growth rate should be analyzed. The dotted line in figure 5 gives the predicted crystallization temperature for $\Delta g_{\mathrm{c}}=\Delta g_{\mathrm{c}}(\mathrm{sd}) / 2$, assuming the maximum variation of the activation energy for jumping, i.e., $\delta \Delta G_{\mathrm{A}}=\delta \Delta g_{\mathrm{c}}$ (see equation (5)). Consequently, the deviation with respect to the standard kinetics would be lower than that in figure 5, which corresponds to a diminution of the crystallization rate by a half. The activation energy would increase by less than $60 \mathrm{meV}$. Here, the discrepancy with the experiment (if any) is much less serious than when nucleation is considered.

In [13] the epitaxial crystallization rate was found to be independent of the degree of relaxation (i.e., from $\Delta g_{\mathrm{c}}$ ). The authors explained this behavior by considering that the enthalpy in excess of the unrelaxed state was due to structural point defects. They argued that the interface velocity (essentially, $r_{\mathrm{n}}$ ) was dominated by the regions which are less enthalpic between the defects, which would account for most of the a-Si volume. Since our interpretation of the crystallization enthalpy variations from sample to sample relies on varying densities of point defects [10], the argument of [13] could be translated to the absence of any systematic variation of the growth rate of our samples.

\section{Summary}

The classical theory of nucleation has been reviewed in order to deduce the dependence of the crystallization kinetics (activation energies and pre-exponential constants) on the enthalpy of the amorphous state. According to this theory, it has been shown that a strong dependence is expected through the nucleation barrier whereas the barrier for atomic jumping and the growth rate are much less sensitive. These predictions have been tested with the help of calorimetric experiments conducted on a large series of a-Si samples that allow simultaneous determination of the crystallization enthalpy, $\Delta h_{\mathrm{c}}$, and the crystallization kinetic parameters. Given that $\Delta h_{\mathrm{c}}$ values expand with a factor of over 2 , the theory predicts that crystallization should occur in a temperature range as wide as $550^{\circ} \mathrm{C}$ and that the activation energy should vary by $40 \%$ within the set of samples. No such large variations are found, but all of the samples crystallize within a narrow range of crystallization rates. This discrepancy is addressed by considering that a-Si is inhomogeneous, so different crystallization enthalpies are compatible with the existence of microscopic domains that are virtually identical for all samples. These domains, where nucleation takes place, would be characterized by the absence of defects and maximum values of the short-range (SRO) and medium-range order (MRO). Independent Raman and XRD experiments indicate that both SRO and MRO in all samples tend to a common 
value independently of the crystallization enthalpy. It is argued that the SRO and the MRO determine the free energy and the a-c interfacial energy of these microscopic domains, respectively.

In principle, our analysis involving the MRO and SRO concepts used here could be directly applied to other tetrahedral covalent amorphous materials (like Ge or GaAs). In more general terms, the role played by microscopic inhomogeneities in the crystallization kinetics could also apply to other amorphous materials and glasses. This makes it much more difficult to achieve the goal of modeling measurements of the nucleation rate or crystal growth velocity from the values of macroscopic thermodynamic magnitudes [44].

\section{Acknowledgments}

This work was partially funded by the Spanish MICINN (MAT2006-11144 project and SB2006-0062 post-doctoral contract) and by the Generalitat de Catalunya (Contract Nos 2009SGR-185, 2009-SGR-770).

\section{References}

[1] Spinella C, Lombardo S and Priolo F 1998 J. Appl. Phys. 845383

[2] Shi G and Seinfeld J H 1991 J. Mater. Res. 62091

[3] Shi G and Seinfeld J H 1991 J. Mater. Res. 62097

[4] Iverson R B and Reif R 1987 J. Appl. Phys. 621675

[5] Farjas J, Roura P and Roca i Cabarrocas P 2007 Mater. Res. Soc. Symp. Proc. 989 A06-17

[6] Mahan A H, Ahrenkiel S P, Roy B, Schropp R E I, Li H and Ginley D S 2008 Thin Solid Films 516529

[7] Nakazawa K and Tanaka K 1990 J. Appl. Phys. 681029

[8] Mahan A H, Su T, Williamson D L, Gedvilas L M, Ahrenkiel S P, Parilla P A, Xu Y and Ginley D A 2009 Adv Funct. Mater. 192338

[9] Young D L, Stradins P, Iwaniczko E, To B, Reedy R C, Yan Y, Branz H M, Lohr L, Alvarez M, Booske J, Marconnet A and Wang Q 2005 Mater. Res. Soc. Symp. Proc. 862233

[10] Kail F, Farjas J, Roura P, Secouard C, Nos O, Bertomeu J and Roca i Cabarrocas P 2011 Phys. Status Solidi RRL 5361

[11] Kelton K F, Greer A L and Thomson C V 1983 J. Chem. Phys. 796261

[12] Turnbull D and Fisher J C 1949 J. Chem. Phys. 1771
[13] Roorda S and Lavigueur Y 2010 Phil. Mag. 903787

[14] Koga N, Nakagoe Y and Tanaka H 1998 Thermochim. Acta 318239

[15] Koga N and Yamane Y 2008 J. Therm. Anal. Calorim. 94379

[16] Radha A V, Forbes T Z, Killian ChE, Gilbert P U P A and Navrotsky A 2010 Proc. Natl Acad. Sci. 2116438

[17] Guo F Q and Lu K 1998 Phys. Rev. B 5710414

[18] Tani Y, Shirakawa Y, Shimosaka A and Hidaka J 2001 J. Non-Cryst. Solids 293779

[19] Al-Ghamdi A A, Alvi M A and Khan A 2011 J. Alloys Compounds $\mathbf{5 0 9} 2087$

[20] Naqvi S F, Deepika, Saxena N S and Bhandari D 2011 Phil. Mag. Lett. 91189

[21] Sun N X, Lu K and Jiang Q 1997 Phys. Rev. B 565885

[22] Donovan E P, Spaepen F, Turnbull D, Poate J M and Jacobson D C 1985 J. Appl. Phys. 571795

[23] Roura P, Sanchez-Rodriguez D and Farjas J 2011 Thermochim. Acta $\mathbf{5 2 2} 161$

[24] Saadane O, Lebib S, Kharchenko A V, Longeaud C and Roca i Cabarrocas P 2003 J. Appl. Phys. 939371

[25] Kail F, Farjas J, Roura P, Seccouard C, Nos O, Bertomeu J, Alzina F and Roca i Cabarrocas P 2010 Appl. Phys. Lett. 97031918

[26] Avrami M 1939 J. Chem. Phys. 71103

[27] Avrami M 1940 J. Chem. Phys. 8212

[28] Avrami M 1940 J. Chem. Phys. 9177

[29] Johnson W A and Mehl R F 1939 Trans. Am. Inst. Mining Met. Eng. 135416

[30] Kolmogorov A N 1937 Izv. Akad. Nauk SSSR, Ser. Fiz. 1355

[31] Farjas J and Roura P 2006 Acta Mater. 545573

[32] Farjas J, Rath C, Roura P and Roca i Cabarrocas P 2004 Appl. Surf. Sci. 238165

[33] Kissinger H E 1957 Anal. Chem. 291702

[34] Roura P and Farjas J 2009 J. Mater. Res. 243095

[35] Roura P, Farjas J and Roca i Cabarrocas P 2009 J. Nanosci. Nanotechnol. 93700

[36] Roura P, Farjas J and Roca i Cabarrocas P 2008 J. Appl. Phys. 104073521

[37] Williamson D L 2003 Sol. Energy Mater. Sol. Cells 7841

[38] Beeman D, Tsu R and Thorpe M F 1985 Phys. Rev. B 32874

[39] Secouard C, Ducros C, Roca i Cabarrocas P, Duffar T and Sanchette F 2007 Proc. 22nd EUPVSEC Milan, p 2036

[40] Dixmier J 1992 J. Physique I 21011

[41] Voyles P M and Abelson J R 2003 Sol. Energy Mater. Sol. Cells 7885

[42] Lebib S and Roca i Cabarrocas P 2004 Eur. Phys. J. Appl. Phys. 2617

[43] Nguyen-Tran T, Suendo V, Roca i Cabarrocas P, Nittala L T, Bofle S N and Abelson J R 2006 J. Appl. Phys. 100094319

[44] Kalb J, Spaepen F and Wuttig M 2003 J. Appl. Phys. 932389 